\title{
Synchrotron x-ray study of melting in submonolayer Ar and other rare-gas films on graphite
}

\author{
J. P. McTague* \\ Chemistry Department, University of California, Los Angeles, California 90024
}

J. Als-Nielsen, J. Bohr, and M. Nielsen

Physics Department, Risø National Laboratory, DK-4000 Roskilde, Denmark

(Received 4 January 1982)

\begin{abstract}
Synchrotron X-ray diffraction studies of the (10) peak of Ar on the (001) surface of $Z Y X$ graphite show a sharp but continuous broadening of the Bragg peak with increasing temperature. Below a coverage of $\sim 1$ Ar atom per six surface carbon atoms $(\rho=1)$ the onset of this transition occurs at a coverage-independent temperature $T=47.9 \mathrm{~K}$. We interpret this point as a critical end point for melting. For coverages $0.94<\rho<1.2$ continuous broadening is also observed, but the onset occurs at a temperature which increases approximately linearly with coverage. This behavior differs markedly from that of other rare-gas films on graphite.
\end{abstract}

\section{INTRODUCTION}

Melting in two dimensions (2D) is intriguing for several reasons. Prime among these is the tendency for transitions to be weaker in lower dimensions, giving hope that the nature of melting and freezing (at least in 2D) can be determined by observing precursor fluctuations in the transition region. Indeed, model calculations ${ }^{1,2}$ indicate that, under some circumstances, the transition may be so weakened as to completely suppress the first-order character always observed in 3D. Of further interest is the staggered "external fields" from the periodic potential of a substrate crystal, which can lead to orientational epitaxy ${ }^{3}$ and modulated structures in some incommensurate systems, as well as to commensurate-incommensurate transitions in the 2D solid. ${ }^{4-6}$ The solid-fluid transition in systems which have commensurate phases has been the subject of much fruitful experimental ${ }^{7-10}$ and theoretical study. ${ }^{11,12}$

Our main purpose in this work is to use $\mathrm{x}$-ray diffraction to explore, at high resolution and surface homogeneity, the melting transition in submonolayer incommensurate Ar film on graphite and to compare the behavior with that of a commensurate film $\mathrm{Kr}$ and another incommensurate one $\mathrm{Xe}$ at similar coverage. The $\mathrm{Kr}$ and $\mathrm{Xe}$ films have already been examined in some detail by Birgeneau et al., ${ }^{9}$ Moncton et al.,${ }^{13}$ and Heiney et al. ${ }^{14}$ As we shall see, comparison of all three systems under the same experimental conditions is of significant aid in developing an overall picture of $2 \mathrm{D}$ phase behavior.

Submonolayer argon films have been explored by several techniques but it cannot be said that a coherent interpretation of the results has emerged. Heat capacity ${ }^{15}$ and neutron diffraction ${ }^{16}$ studies on a Grafoil substrate (surface coherence length $L \approx 120 \AA$ ) show broad features in the region $40<T<60 \mathrm{~K}$, which suggest continuous transition behavior. However, the possibility of rounding due to finite-size effects in these measurements must be considered. Low-energy electron diffraction (LEED) work on a single crysta ${ }^{17}$ points to a rather sharp transition, as do adsorption isotherm data ${ }^{18}$ on uncompressed exfoliated graphite. The isotherm data, however, have been interpreted in terms of a registered 2D solid, in contradiction with direct structural determinations. Some computer simulations have been interpreted in terms of continuous melting, ${ }^{19}$ while others ${ }^{20,21}$ have asserted first-order behavior.

Moncton et al. ${ }^{13}$ and Birgeneau et al..$^{9}$ have shown that high-resolution synchrotron $x$-ray diffraction from rare-gas films on $Z Y X$ graphite $(L \approx 2000 \AA)$ can provide unprecedented detail concerning thin-film structure and phase transitions. We have confirmed the large length scale, ${ }^{22}$ and here report studies on Ar films at coverages $0.94<\rho<1.2$ in units where $\rho=1$ corresponds to one atom per three surface carbon hexagons 


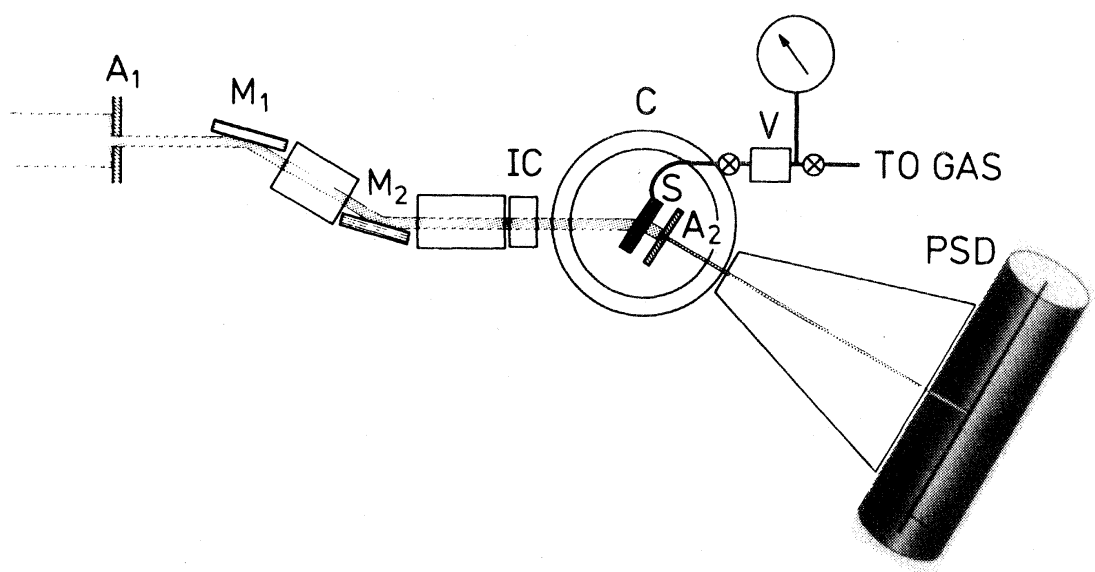

FIG 1. Schematic top view of setup. $A_{1}: 4 \times 6 \mathrm{~mm}^{2}$ slit, $A_{2}: 1 \times 6 \mathrm{~mm}^{2}$ slit. $M_{1}$ and $M_{2}: \operatorname{Ge}(111)$ monochromators. IC: Ionization chamber monitor. $C$ : Cryostat. $S$ : Graphite sample cell. $V$ : Standard volume with pressure gauge. PSD: Position-sensitive detector. Vacuum beam-path with Be windows between $M_{1}-M_{2}, M_{2}-I C$, and $C-$ PSD.

$(\sqrt{3} \times \sqrt{3}$ registered coverage). Comparative studies of $\mathrm{Kr}$ and $\mathrm{Xe}$ at submonolayer coverages help to calibrate the system variables (rounding effects, absolute coverage, etc.) and, as we shall see, provide a striking contrast to the Ar results.

\section{EXPERIMENTAL}

The experiment was carried out at the storage ring DORIS, operating at $3.3 \mathrm{GeV}$ and a typical current of $50 \mathrm{~mA}$. The synchrotron radiation at beamline $D 4$ (see Fig. 1) has a horizontal aperture of $1 \mathrm{mrad}$, but as we do not presently have any focusing elements installed, we only utilize a small fraction of this aperture. A monochromatic beam is extracted from the synchrotron beam by Bragg reflection from a perfect $\mathrm{Ge}$ crystal in the (111) orientation. Second order is thus forbidden but third order and higher harmonics are a source of beam contamination. It is easily seen that thirdorder Bragg reflection from (110) of the graphite honeycomb lattice gives exactly the same Bragg angle as first-order Bragg reflection from the $\sqrt{3}$ commensurate physisorbed layer, and it is therefore important to be able to eliminate the higher-order contamination. This is done by a second monochromator crystal identical to the first but misset about one Darwin width of the (111) reflection. In that case most of the first order is transmitted but higher harmonics are eliminated partly because their Darwin widths are smaller and partly because the Bragg angles are slightly different due to the different refractive index for first order and the higher harmonics.

The monochromatic beam hits the graphite substrate (Union Carbide $Z Y X$ ) consisting of flakes with a common vertical $c$ axis, and the scattered radiation is detected by a position-sensitive detector PSD. ${ }^{23}$ The resolution is essentially determined by the width of the sample seen from the PSD. We used a 1-mm slit positioned inside the sample cryostat and adjacent to the sample and a PSD distance of $600 \mathrm{~mm}$, so the resolution corresponds to a Gaussian coherence length of $1200 \AA$, of the same order of magnitude as the intrinsic graphite coherence length of $1600 \AA$ determined by a triple-axis measurement in an earlier experiment. ${ }^{22}$

The graphite substrate, initially baked out at $800^{\circ} \mathrm{C}$, is contained in a sealed cell with Be windows. The cell is connected via a capillary to a standard filling volume and a pressure gauge outside the Displex cryostat. The amount of gas giving on adsorption a complete registered physisorbed monolayer $(\rho=1)$ was determined by $\mathrm{x}$ ray diffraction profiles on $\mathrm{Kr}$ at $T=10 \mathrm{~K}$. At completion of a monolayer, $\mathrm{Kr}$ undergoes a firstorder transition to a modulated-domain structure and $\rho=1$ is defined as the filling at which the domain-structure pattern begins to appear. The accuracy of this method is about $2 \%$. A secondary standard is provided by accurately measured $\mathrm{Ar}$ isotherms. During the experiment we monitored the isotherm several times to ensure that the available area for physisorption remained constant.

In Fig. 2 we show the thermal behavior of the (10) diffraction peak of $\mathrm{Xe}(\rho=0.61)$ and $\mathrm{Kr}$ $(\rho=0.73)$. Since the incommensurate Xe lattice 


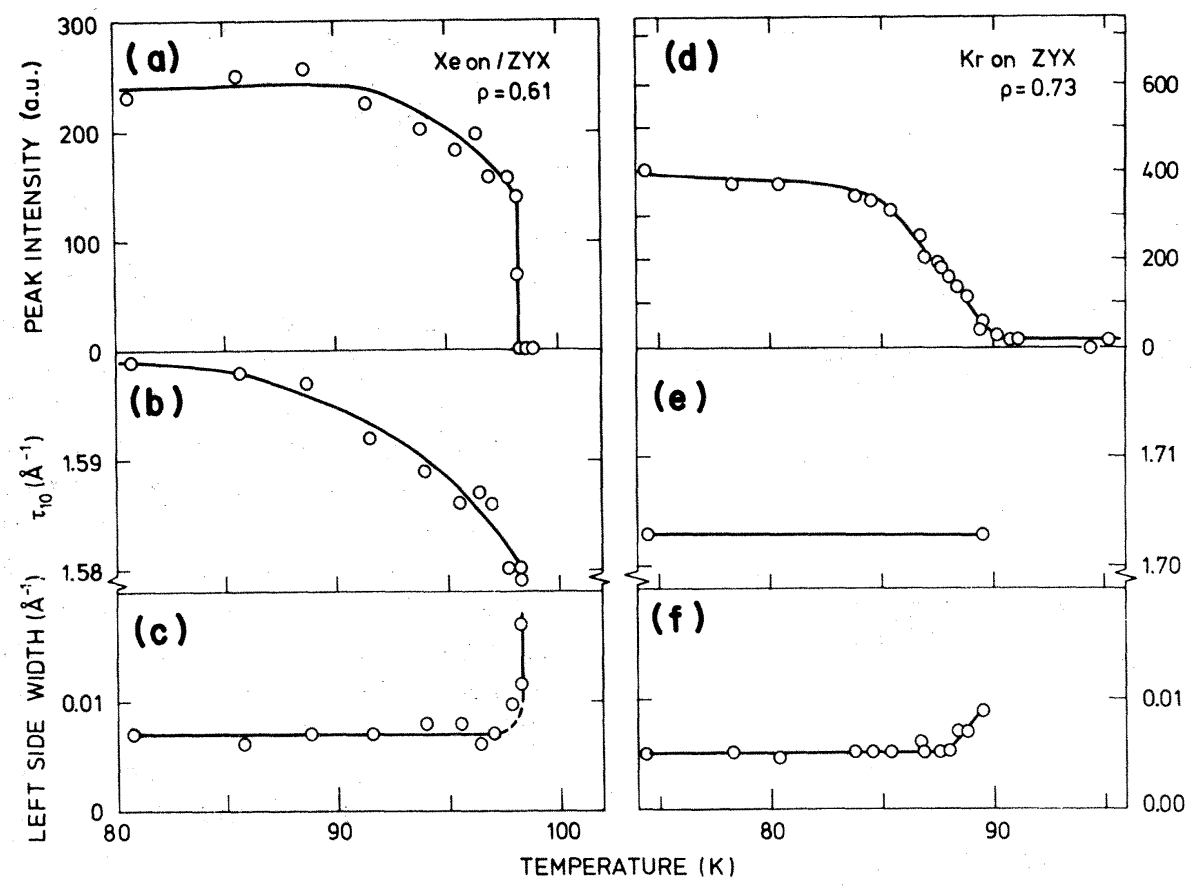

FIG. 2. (10) Bragg peak disappearance with increasing temperature for adsorbed submonolayers of $\mathrm{Xe} \mathrm{Kr}$ on graphite. $a$ and $b$, and $d$ and $e$ show intensities and positions of the Bragg peak, $c$ and $d$ the width of their left-hand side. The curves are guides to the eye only. These transitions are denoted by $X$ in the phase diagrams of Fig. 6 .

constant is about $8 \%$ larger than that of the commensurate $\mathrm{Kr}$ lattice, both coverages correspond to about $73 \%$ of a complete monolayer. For Xe the peak intensity drops precipitously over a temperature range of no more than $0.02 \mathrm{~K}$, centered at $T_{M}=98.25 \mathrm{~K}$. There is no peak broadening below $T=97.5 \mathrm{~K}$, and only a minor change above there. These results are in agreement with conclusions from adsorption isotherms ${ }^{7}$ and heat capacity ${ }^{8}$ that there is a $2 \mathrm{D}$ triple point (solid-liquid-gas) for $\mathrm{Xe}$, implying that in the range of our observations the $s-l, s-g$, and $l-g$ transitions are all of first order. Both liquid and gas diffraction patterns are too broad to be seen under our experimental conditions. Our results also agree with the $\mathrm{x}$-ray work of Birgeneau et al. ${ }^{9}$ Thus 2D Xe seems to be quite analogous to $3 \mathrm{D} \mathrm{Xe}$, although the peak intensity shows significant percursor behavior on the solid side. Note that at the triple point, the Xe nearestneighbor distance is some $8 \%$ expanded from registry $\left(\tau_{10}^{\mathrm{reg}}=1.703 \AA^{-1}\right)$.

In contrast, the $\mathrm{Kr}$ film remains registered at this coverage until the solid peak intensity vanishes [see Fig. 2(e)]. Likewise, the solid maintains a substrate-limited correlation length until almost all Bragg intensity has gone [Fig. 2(f)]. There is an approximately linear peak-intensity decrease over the temperature range $85<T<90 \mathrm{~K}$ [Fig. 2(d)].

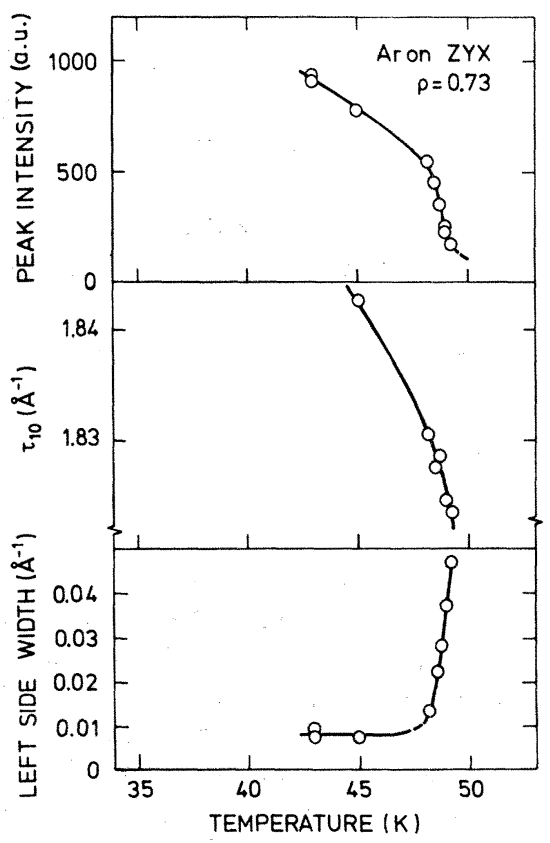

FIG. 3. Peak intensity, position, and width of the left side of the (10) Bragg peak from Ar submonolayers on graphite. The curves are guides to the eye only. 

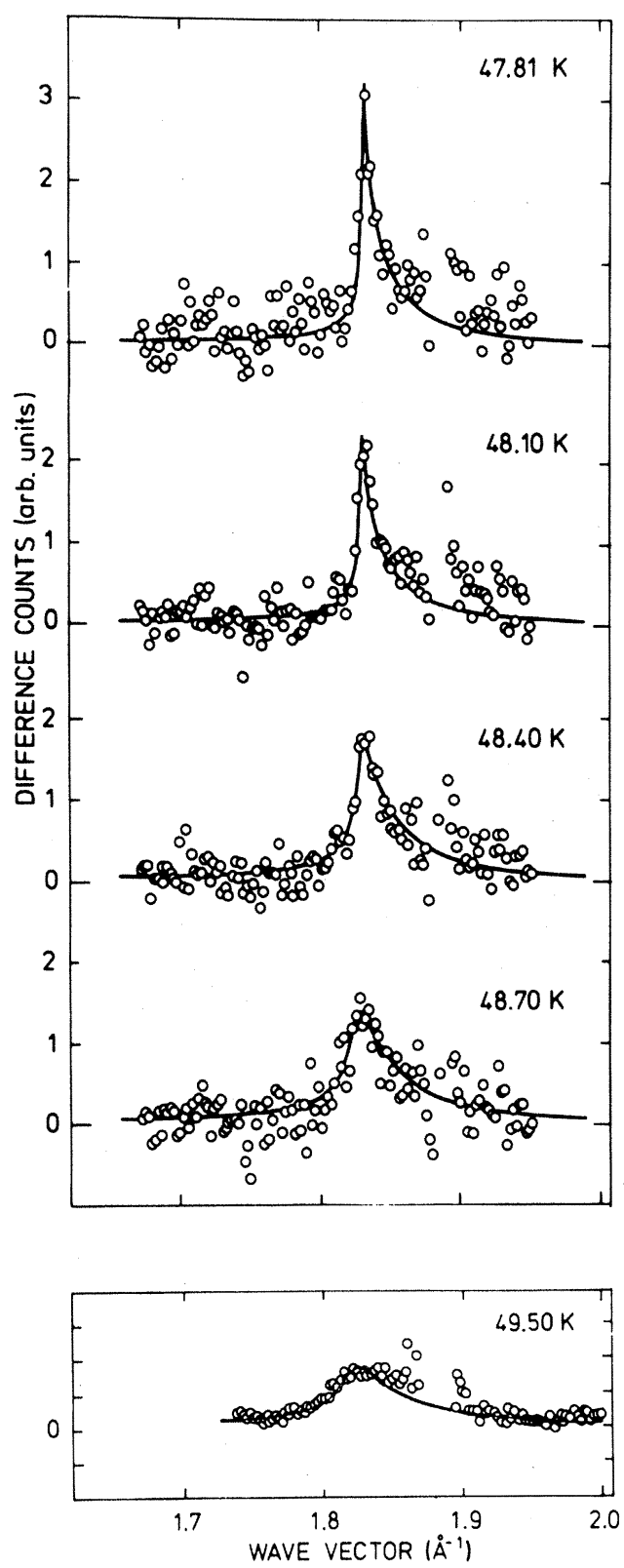

FIG. 4. Measured Bragg profiles at the (10) reflection from Ar monolayers on graphite. The upper four panels show the results obtained with synchrotron $\mathrm{x}$-rays and with the average Ar layer density $\rho=0.83$. The lower-panel data were measured with a rotating $x$-ray source and with $\rho=0.93$. The curves are calculated line shapes using a powder-and-tilt averaged Lorentzian scattering function (Ref. 14) with correlation lengths $\xi$ given by formula, 1 . $T_{M}=47.9 \mathrm{~K}, \xi=1800,1065,306$, $187,104 \AA$. $\xi$ vs $T$ is also shown in Fig. 5. Data near $Q=1.8 \AA^{-1}$ are distorted by imperfect graphite (002) background subtraction.
These results imply a first-order-registered soliddisordered fluid phase transition which occurs over a significant temperature range at constant coverage, due to following the melting line as a function of surface spreading pressure, which increases as the melted fraction increases, cf. Fig. 6(b). The existence of a melting range implies that there is no triple point, and thus no three-phase coexistence (no 2D liquid-gas coexistence) for this system.

The first-order nature of the registered $\mathrm{Kr}$ transition is in agreement with heat capacity ${ }^{8}$ and adsorption isotherm data, ${ }^{7}$ as well as X-ray diffraction at higher coverage ${ }^{9}$ and molecular dynamics simulations ${ }^{12}$ which indicate that the disordered phase is fluid. The $\mathrm{x}$-ray results imply that along the melting line, the fluid is correlated over only short distances, as typical for 3D fluids.

The melting of (incommensurate) Ar is quite different from that of both incommensurate $\mathrm{Xe}$ and commensurate Kr. Results for $\rho=0.73$ and 0.83 are summarized in Figs. 3 and 4. In the melting region the Ar nearest-neighbor distance is $8 \%$ smaller than the $\sqrt{3}$ registered spacing, yet the properties do not resemble those of Xe $8 \%$ larger than the $\sqrt{3}$ spacing). The (10) peak intensity decreases significantly over the range $43<T \leqq 48 \mathrm{~K}$, then plunges sharply but continuously over the next degree or so in a manner indicative of a higher-order phase transition. Likewise, the peak width, which is essentially constant for $T<48 \mathrm{~K}$, increases rapidly but continuously between $T=47.9$ $\mathrm{K}$ and $T=50 \mathrm{~K}$. Essentially identical behavior was observed over the range $0.73 \leq \rho<+0.94$, including the onset of peak broadening at $T=47.9$ $\mathrm{K}$. Without high-resolution data, this invariance of the onset point might be interpretable as a triple point. However, all our data point to a continuous peak broadening for Ar.

Comparison of our $\mathrm{x}$-ray structural data with the heat capacity results of Chung ${ }^{15}$ on a similar coverage of Ar on Grafoil offers interesting clues concerning the melting mechanism. In Fig. 5 we show the heat capacity data of Chung for a $\rho$ $=0.59$ film of Ar on Grafoil. Note that the heat capacity is quite broad, with a maximum near $50-51 \mathrm{~K}$, close to but significantly above the point $(47.9 \pm 0.2 \mathrm{~K})$ where $S(Q)$ begins to broaden in our $Z Y X$ data. Although it is conceivable that this temperature difference could be due to finitesize effects in Grafoil $(L \sim 120 \AA)$ as compared with $Z Y X(L \sim 1600-2000 \AA)$, such a result is in fact qualitatively predicted by the KosterlitzThouless, ${ }^{1}$ Halperin-Nelson ${ }^{2}$ dislocation theory of melting for the case of dilute dislocation pairs at 


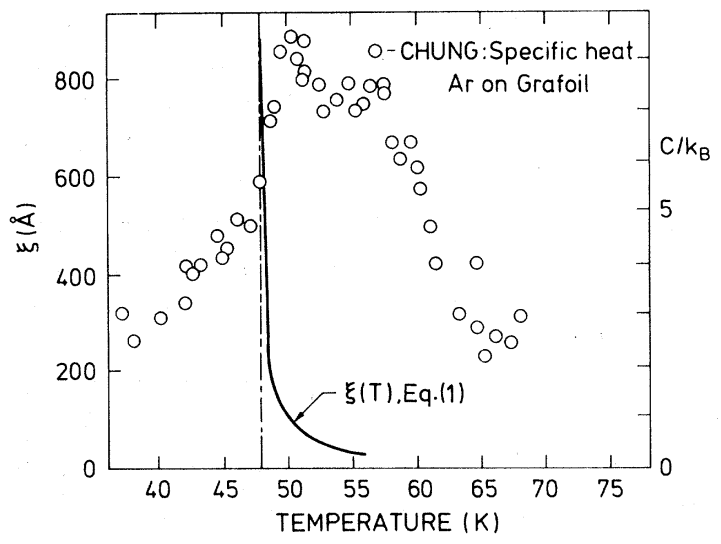

FIG. 5. Points show the specific-heat data of Chung (Ref. 15) for Ar monolayers on graphite, $\rho=0.59$. The values of specific heat $c$ are given by the right-side scale of the diagram. The curve is calculated by formula and show the Lorenzian correlation length $\xi$ vs temperature for the melting temperature $T_{m}=47.9 \mathrm{~K}$ and the constants given by $b=0.576$ and $\xi_{0}=13.8 \AA$. The $\xi$ values of this curve are used in calculating the Bragg profiles of Fig. 4.

the transition. In this case the melting is initiated by the unbinding of a few very large-spacing dislocation pairs. The heat-capacity peak, predicted to be rounded rather than cusplike, corresponds to the unbinding of the much more numerous small dislocation pairs, and occurs at high temperature.

Another prediction ${ }^{2}$ is that on the fluid side of the transition the pair-correlation length $\xi$ should diverge with the unusual form

$$
\xi=\xi_{0} \exp \left[b /\left[\frac{T}{T_{m}}-1\right]^{0.369 \cdots}\right] .
$$

The curve for $T_{m}=47.9 \mathrm{~K}, b=0.576$, and $\xi_{0}=13.8$ $\AA$ is also shown in Fig. 5. Line shapes calculated from this form, using a powder-and-tilt average Lorentzian scattering function, ${ }^{10}$ are shown with the experimental data in Fig. 4. Clearly, the data are compatible with such a form, although the low signal-to-noise ratio does not allow a critical test. However, taken together with the heat capacity, there is a strong suggestion that the melting in $\mathrm{Ar}$ thin films follows the form predicted by the Kosterlitz-Thouless, Halperin-Nelson dislocation melting theory.

It is instructive to compare the phase diagrams of $\mathrm{Ar}, \mathrm{Kr}$, and $\mathrm{Xe}$ on graphite. In 3D these diagrams are essentially identical. For example the ratio between the critical temperature and the triple-point temperature $T_{c}(3 \mathrm{D}) / T_{\mathrm{TP}}(3 \mathrm{D})=1.80$ for $\mathrm{Ar}, \mathrm{Kr}$, and $\mathrm{Xe}$. This is an example of the well- known principle of corresponding states, which results from the approximate scaling properties of the rare-gas pair potentials:

$$
V(r) / \epsilon=f(r / \sigma) .
$$

Here $\epsilon$ is the characteristic energy parameter for the given rare gas and $\sigma$ is the characteristic length scale (such as the atomic diameter). The universal form of the pair potential is given by $f(r / \sigma)$. The experimental 2D diagrams are shown in Fig. 6, where temperatures have been reduced by the respective $3 \mathrm{D}$ triple-point temperatures 84,116 , and $161 \mathrm{~K}$ for $\mathrm{Ar}, \mathrm{Kr}$, and $\mathrm{Xe}$, respectively.

Xe [Fig. 6(a)] has a 2D gas-liquid-incommensurate solid triple point at $T^{*}=0.61$, and adsorption isotherm data ${ }^{7}$ have been interpreted in terms of a gas-liquid critical point near $T^{*}=0.73(=117$

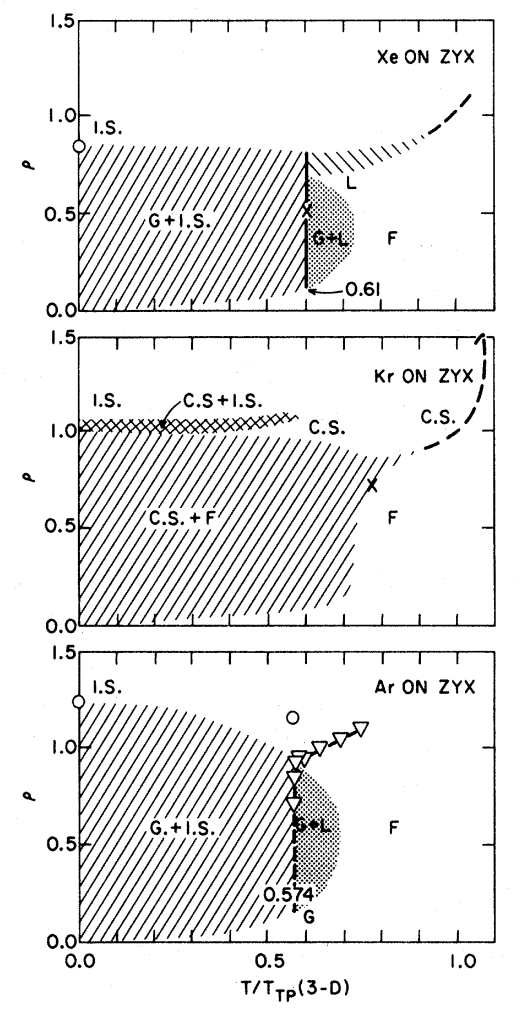

FIG. 6. The phase diagrams for $\mathrm{Xe}, \mathrm{Kr}$, and $\mathrm{Ar}$ monolayers on graphite. The symbols are: $\rho$ : averaged layer density in the units of the density of the $\sqrt{3} \times \sqrt{3}$, $30^{\circ}$ commensurate structure. $G: 2 \mathrm{D}$ gas, $F: 2 \mathrm{D}$ fluid, $L$ : 2D liquid. IS: Incommensurate solid, CS: 2D commensurate solid $\left(\sqrt{3} \times \sqrt{3}, R 30^{\circ}\right)$, and $T / T_{\mathrm{TP}}(3 \mathrm{D})$ : the temperature in units of the triple-point temperatures of the respective 3D systems: The data of Fig. 2 determine the transition points indicated by $X$ for $\mathrm{Xe}$ and $\mathrm{Kr}$. For Ar the density of an ideal 2D solid (without vacancies) as given by the measured position of the (10) Bragg peak at the vertical melting line and at low $T$ is shown by open circles. 
$\mathrm{K})$. Thus, in the submonolayer region the $2 \mathrm{D} \mathrm{Xe}$ phase diagram is quite analogous to the bulk 3D one, although the liquid range (triple point to critical point) is considerably narrower,

$$
T_{c}(2 \mathrm{D}) / T_{\mathrm{TP}}(2 \mathrm{D})=1.20 .
$$

Slightly above one monolayer, however, Heiney et al. ${ }^{14}$ have recently shown that the melting transition becomes continuous rather than first order. This is schematically shown by the dashed line in Fig. 6(a).

The phase diagram for $\mathrm{Kr}$ [Fig. 6(b)], on graphite is quite different from $\mathrm{Xe}$, as is to be expected from the fact that submonolayer $\mathrm{Kr}$ is commensurate with the graphite (001) surface, forming a $\sqrt{3} \times \sqrt{3}, R 30^{\circ}$ structure. As reported above, the melting is first order below $\rho=0.8$ but occurs at temperatures which are coverage dependent. The melting point reported here $\left(T_{m}^{*}=0.78 \sim 90 \mathrm{~K}\right.$, $\rho=0.73)$ is shown in Fig. $6(\mathrm{~b})$ as an $x$. The rest of the phase diagram is taken from Refs. 8,14 , and 24. At high coverages the melting becomes continuous, as indicated by the dashed line. Although the low-temperature, low-coverage solid phase is commensurate, it can be driven incommensurate by either pressure ${ }^{22}$ or coverage. ${ }^{13}$ This commensurate-incommensurate transition has been shown to be first order for low temperatures, ${ }^{22,24}$ but becomes less strong at high temperatures, ${ }^{13}$ and here the order is uncertain. Placing the 2D phase diagram on a reduced temperature scale indicates immediately why $\mathrm{Kr}$ on graphite has no triple point, and thus no liquid-gas phase separation. The commensurate substrate field gives an extra stabilization to the solid and raises the melting point from $T^{*} \sim 0.6$ to $T^{*} \sim 0.7$. Thus, the fluid formed at melting is just above the 2D gas-liquid critical point. ${ }^{8}$ Molecular dynamics calculations ${ }^{12}$ have shown that here the $\mathrm{Kr}$ fluid has only very little correlation with the substrate potential.

Ar on graphite differs qualitatively from $\mathrm{Kr}$ and $\mathrm{Xe}$ in that melting is always continuous, at least up to $\rho=1.2$ Below $\rho=0.94$, the onset occurs at $T=47.9 \pm 0.2 \mathrm{~K}$, the uncertainty being due to the finite grid of data points taken. Above $\rho=0.94$ the onset point increases approximately linearly with coverage $(d T / d \rho \sim 45 \mathrm{~K})$. The invariance of the onset temperature below $\rho-0.94$ indicates that the same solid phase is melting for all such coverages. This requires a first-order gas-solid phase coexistence line up to $T_{m}$. Furthermore, the reported gas-liquid critical point ${ }^{7}$ requires that this firstorder coexistence line continues above $T_{m}$ as a liquid-gas coexistence, terminating at $T_{c}^{*} \sim 0.73$ -0.77 , where the range of $T_{c}^{*}$ indicates various reported values. ${ }^{7}$ Adsorption isotherm data ${ }^{18}$ indicate that $T_{c}^{*} \approx 0.7(=58-59 \mathrm{~K})$. The critical melting line above $\rho=0.94$ terminates in a critical end point at $\left.T^{*}=0.57\right)(=47.9 \mathrm{~K})$. From the known coverage here and the measured position of the (10) Bragg peak $\left(\tau_{10}=1.83 \AA^{-1}\right)$ we can conclude that the solid must have about $20 \%$ vacancies at the solid-liquid critical end point. For reference the density that a perfect solid with $\tau_{10}=1.83 \AA^{-1}$ would have is shown as an open circle in Fig. 6(c). The low-coverage continuous melting point of $T^{*}=0.57$ is close to the first-order triple point of $\mathrm{Xe}\left(T^{*}=0.61\right)$. Likewise, the gas-liquid critical points are close to each other.

\section{DISCUSSION}

The fundamental question posed by these observations is: Why should $\mathrm{Xe}$ and $\mathrm{Ar}$, which at submonolayer coverages are about equally incommensurate with the substrate (one $8 \%$ larger in linear dimension than the $\sqrt{3} \times \sqrt{3}$ structure, the other about $8 \%$ smaller), have qualitatively different melting characteristics, yet melt at almost the same reduced melting point? A corollary question is: Which, if either, represents a close approximation to the properties of a $2 \mathrm{D}$ system on a perfectly smooth substrate?

We are not able to reach definitive answers to these questions but the data reported here, taken together with other information, suggest the following interpretation. The asymmetric shape of the interatomic potential makes it more difficult to compress a film than to expand it from its equilibrium lattice spacing. Thus, for a fixed rare-gassubstrate potential those atoms which naturally have a lattice parameter smaller than that of the registered structure (Ar,Kr) should be more susceptible to the ordering substrate field than larger ones (Xe).

We then assume that $\mathrm{Xe}$ on graphite is a better representation of 2D field-free system than is Ar. Early LEED measurements ${ }^{25}$ of Xe on singlecrystal graphite showed no appreciable orientational ordering of fluid Xe relative to the graphite crystal axes, which is consistent with this assumption. The submonolayer Xe system then is assumed to melt to an essentially isotropic liquid (although there must be a finite degree of ordering in the sixfold substrate field). The transition of a 2D rare-gas solid to an isotropic liquid then appears to 
be first order but with significant precursor behavior on the solid side.

Fluid Ar monolayers, however, have been observed to show significant orientational ordering, ${ }^{17}$ Thus, here the melting transition is solid to oriented liquid ("long-range hexatic"). It has been shown by Halperin and Nelson ${ }^{2}$ that the transition from solid to oriented fluid can be continuous.

Further evidence consistent with this interpretation is provided by the recent synchrotron observation of Heiney et al. ${ }^{14}$ that when Xe is compressed by adding $10 \%$ greater than one monolayer, the melting changes from first order to continuous. Specifically, they find that when $\tau_{10}=1.60 \AA^{-1}$ at melting (versus $\tau_{10}=1.58 \AA^{-1}$ for lower coverages) they observe behavior in quantitative agreement with the Halperin-Nelson fluid line shape and temperature dependence of the fluid-correlation range $\xi$, namely,

$$
\xi=\xi_{0} \exp \left[b /\left(T / T_{m}-1\right)^{0.369 \cdots}\right] .
$$

We assume that the compression has increased the susceptibility of the Xe film to the substrate field sufficiently that its melting then behaves qualitatively like submonolayer Ar. Note that only a small compression, from $8 \%$ expansion down to $6 \%$, produces this qualitative change. Thus, submonolayer Xe must be in some sense close to having continuous melting. In conclusion, we reiterate that, although this interpretation is consistent with available observations, it is by no means proven. This will require high-resolution LEED or $\mathrm{x}$-ray data on single crystals for rare-gas films as a function of compression.

\section{ACKNOWLEDGMENTS}

We wish to thank P. Heiney and collaborators for communicating their results ${ }^{14}$ prior to publication. The hospitality of HASYLAB is gratefully acknowledged. This research was supported in part by the Danish Natural Science Foundation and the U. S. National Science Foundation.
"Present address: National Synchrotron Light Source, Brookhaven National Laboratory, Upton, New York 11973.

1J. M. Kosterlitz and D. J. Thouless, J. Phys. C $\underline{6}, 1181$ (1973).

${ }^{2}$ B. I. Halperin and D. R. Nelson, Phys. Rev. Lett. 41, 121 (1978); D. R. Nelson and B. I. Halperin, Phys. Rev. B 19, 2457 (1979).

${ }^{3}$ A. D. Novaco and J. P. McTague, Phys. Rev. Lett. $\underline{38}$, 1286 (1977).

4J. Villain, in Ordering in Strongly Fluctuation Condensed Matter Systems, edited by T. Riste (Plenum, New York, 1980), p. 221.

${ }^{5}$ H. Shiba, J. Phys. Soc. Jpn. 뜨, 1852 (1979); 48, 211 (1980).

${ }^{6}$ P. Bak, D. Mukamel, J. Villain, and K. Wentowski, Phys. Rev. B 19, 1610 (1979).

${ }^{7}$ A. Thomy and X. Duval, J. Chim. Phys. 66, 1966 (1969); 67, 286 (1970); 67, 1101 (1970).

${ }^{8}$ D. M. Butler, J. A. Litzinger, G. A. Stewart, and R. B. Griffiths, Phys. Rev. Lett. 42, 1289 (1979); J. A. Litzinger and G. A. Stewart, in Ordering in Two Dimensions, edited by S. K. Sinha (Elsevier, New York, 1980), p. 267.

${ }^{9}$ R. J. Birgeneau, E. M. Hammons, P. Heiney, and P. W. Stephens, in Ordering in Two Dimensions, edited by S. K. Sinha (Elsevier, New York, 1980), p. 29; R. J. Birgeneau, G. E. Brown, P. M. Horn, D. E. Moncton, and P. W. Stephens, J. Phys. C. 14, L49 (1981).
${ }^{10}$ S. K. Sinha, in Non-Linear Phenomena at Phase Transitions and Instabilities, edited by T. Riste (Plenum, New York, 1981), p. 433.

${ }^{11}$ A. N. Berker, in Ordering in Two Dimensions, edited by S. K. Sinha (Elsevier, New York, 1980), p. 9.

${ }^{12}$ F. Hanson and J. P. McTague, J. Chem. Phys. 72, 6363 (1980).

${ }^{13}$ D. E. Moncton, P. W. Stephens, R. J. Birgeneau, P. M. Horn, and G. S. Brown, Phys. Rev. Lett. $\underline{46}$, 1533 (1981).

${ }^{14}$ P. A. Heiney, R. J. Birgeneau, G. S. Brown, P. M. Horn, D. E. Moncton, and P. W. Stephens, Phys. Rev. Lett. 48, 104 (1982).

${ }^{15}$ T. T. Chung, Surf. Sci. $\underline{87}, 348$ (1979).

${ }^{16}$ H. Taub, K. Carneiro, J. K. Kjems, L. Passell, and J. P. McTague, Phys. Rev. B 16, 4551 (1977)..

${ }^{17}$ C. G. Shaw, S. C. Fain, Jr., and M. D. Chinn, Phys. Rev. Lett. 41, 955 (1978); C. G. Shaw and S. C. Fain, Jr., Surf. Sci. 83, 1 (1979); 91, L1 (1980).

${ }^{18}$ F. Millot, J. Phys. (Paris) 40, L9 (1979); Y. Larher and B. Gilquin, Phys. Rev. A 20, 1599 (1979).

${ }^{19}$ F. E. Hanson, M. J. Mandell, and J. P. McTague, J. Phys. (Paris) $\underline{38}$, C4-76 (1977).

${ }^{20}$ S. Toxvaerd, Phys. Rev. Lett. 44, 1002 (1980).

${ }^{21}$ F. F. Abraham, Phys. Rev. Lett. 44, 463 (1980).

${ }^{22}$ M. Nielsen, J. Als-Nielsen, J. Bohr, and J. P. McTague, Phys. Rev. Lett. 47, 582 (1981).

${ }^{23}$ It should be noted that it is not possible to pulseheight discriminate against higher-order contam- 
ination with a position-sensitive proportional gas-flow counter, and that is the reason why we had to use a double monochromator in this case. In a standard triple-axis setup where the Bragg profiles are measured point by point using a scintillation detector pulse-height discrimination is in fact sufficient to eliminate higher-order contamination.
24J. Bohr, M. Nielson, and J. Als-Nielsen, in Proceedings of Third European Conference on Surface Science, Paris, 1980, edited by D. A. Degras and M. Costa (Societé Française du Vide, Paris, 1980), Vol. 1, p. 112.

${ }^{25}$ J. J. Lander and J. K. Morrison, Surf. Sci. $\underline{6}, 1$ (1967). 


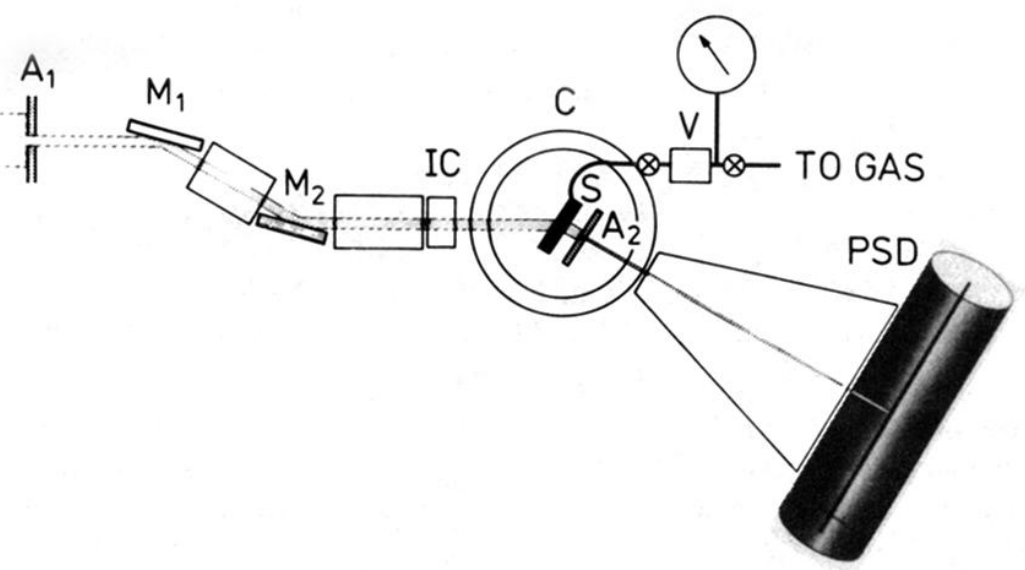

FIG 1. Schematic top view of setup. $A_{1}: 4 \times 6 \mathrm{~mm}^{2}$ slit, $A_{2}: 1 \times 6 \mathrm{~mm}^{2}$ slit. $M_{1}$ and $M_{2}: \mathrm{Ge}(111)$ monochromators. IC: Ionization chamber monitor. $C$ : Cryostat. $S$ : Graphite sample cell. $V$ : Standard volume with pressure gauge. PSD: Position-sensitive detector. Vacuum beam-path with Be windows between $M_{1}-M_{2}, M_{2}-I C$, and $C-$ PSD. 